\title{
EL PROYECTO DOCENTE EN LA UNIVERSIDAD ESPAÑOLA SEGÚN EL ESPACIO EUROPEO DE EDUCACIÓN SUPERIOR
}

\section{Introducción}

La Ley Orgánica 4/2007 del 12 de abril (Boletín Oficial del Estado, BOE del 13 de abril de 2007) se justifica porque los países de la Unión Europea y del entorno iniciaron un movimiento para la creación de un ámbito de educación universitaria superior común basado en la movilidad, el reconocimiento de las titulaciones y la formación a lo largo de la vida (BOE, 307, 49400-49403); lo que supuso un cambio muy importante al atribuirle a la universidad un rol fundamental en el desarrollo de la sociedad española, no solo en el ámbito económico, sino también en el de la condición humana, ya que no en vano fue concebida como un servicio público.

Además, el papel del profesor sufrió un profundo cambio para propiciar la adquisición de competencias por parte de los alumnos (De Miguel, 2006; González, 2010). El énfasis de su actividad residió en su capacidad para diseñar situaciones y dar respuesta a las necesidades educativas, implementando sistemas de tutorías y seguimiento del proceso de aprendizaje, con lo cual contribuir a una formación integral de los futuros profesionales de la enseñanza (Knight, 2005).

\section{El proyecto docente en el Espacio Europeo de Educación Superior (EEES)}

Concebimos el proyecto docente como un documento que expresa los propósitos que surgen de la interacción entre el profesorado y una materia con el punto de mira puesto en un alumnado concreto. Fruto de la reflexión, la iniciativa marca un camino que recoge una filosofía y, por lo tanto, está sujeta a las variaciones inspiradas por las características concretas en las que se va desarrollando el acto educativo. Dicho proyecto está sostenido sobre tres pilares: un marco 
legislativo, un marco conceptual y una trayectoria vital por parte del docente que incluye su experiencia profesional, su formación (inicial y continua) y su experiencia personal (Malheiro, 2011).

Como implicados en este proceso, somos conscientes de que nos encontramos delante de una difícil tarea -posiblemente la más comprometida con el mundo- en la que es preciso que seamos conocedores en profundidad de nuestro oficio, así como también capaces de encontrar las soluciones más adecuadas a los problemas complejos que plantea la educación (Levy-Leboyer, 2000). El cambio de paradigma en los procesos formativos que ha supuesto el diseño de los planes de estudio, al pasar de un diseño por directrices a uno basado en competencias, ha motivado también un cambio de modelo en los procesos de enseñanza-aprendizaje y, por lo tanto, en los procesos evaluativos, lo que ha supuesto lógicamente un reto para el profesorado al tener que aplicar nuevas metodologías evaluativas para asegurar la adquisición de las competencias (Muñoz, Rebollo y Espiñeira, 2013). Sin embargo, estamos aún en una etapa inicial del proceso en el que, como señalan Triadó, Aparicio y Elasri (2013), "la metodología que facilite el cambio cultural hacia el desarrollo y evaluación de competencias está por descubrirse en la mayoría de universidades y grados" (p. 35). Dicha complejidad se debe en parte a la modificación del papel del alumnado, como señalan Delgado, Borge, García, Oliver y Salomón (2005), o bien Montero (2010), ya que ahora el aprendizaje no solo está centrado en la adquisición de conocimientos, sino que el estudiante también debe actuar como motor de su aprendizaje ante cualquier situación o experiencia educativa a lo largo de toda su vida.

Por nuestra parte la reivindicación de la profesionalidad no podía quedar limitada a actos técnicos de la función docente, sino que debíamos plantear un desarrollo profesional más amplio, una nueva cultura profesional que propiciara espacios de reflexión y crítica respecto de las funciones de la educación, del modelo de sociedad y proyecto de ser humano, los procesos de formación, las condiciones laborales y las variables del proceso de enseñanzaaprendizaje, teniendo como objetivo "el profesor investigador de su práctica profesional” (Imbernon, 2000; Fernández-Salinero, 2006). 


\section{Origen y evolución del Espacio Europeo de Educación Superior}

A fin de presentar un artículo que resulte lo más claro posible, exponemos a continuación el origen y la evolución del EEES, estructurador de la nueva acción educativa en la universidad, del desarrollo de las materias -la guía docente es el referente básico- y de su currículo.

Debido a la incorporación de España al entorno europeo y mundial, especialmente en la Comunidad Económica Europea, CEE, y la Organización del Tratado del Atlántico Norte, OTAN, surgieron numerosas voces que incitaban a la necesidad de una reforma universitaria que permitiera "adaptar" la universidad española al "progreso" mundial que se estaba viviendo en las últimas décadas.

Con motivo de la celebración del 750 aniversario de la Universidad de la Sorbona de París en 1998, tuvo lugar una primera Declaración de los ministros de Educación Superior de Francia, Alemania, Reino Unido e Italia, donde se apuntalaron los primeros acuerdos para iniciar un proceso de convergencia europea en materia educativa en la enseñanza superior, lo que transformaría la universidad de muchos países de Europa. Como rúbrica de este principio de acuerdo se firmó la Declaración de la Sorbona (1998), cuya idea subyacente era la creación de un espacio común de conocimientos, pero con autonomía en cada universidad, facultad, departamento y profesor. En 1999 se materializó la Declaración de Bolonia, en la que se determinó el establecimiento de un sistema europeo de educación superior antes de 2010.

En este contexto se le atribuye a la universidad un rol fundamental en el impulso del desarrollo de una nueva sociedad, que basa su progreso en el conocimiento y se dedica a la formación, a la educación, a la investigación y a la transferencia de la tecnología de su contorno. Así mismo, se promueve una reforma educativa que permite hablar de una Europa que, desde el punto de vista económico, haga a la universidad más competitiva y dinámica en el mundo, y sea un referente de calidad mundial (Villa y Villa, 2007). El éxito de 
este posicionamiento, en principio, radica en conseguir la excelencia universitaria por medio de un enfoque interdisciplinar que busque la competencia y la idoneidad de los profesionales, tanto desde el punto de vista del conocimiento conceptual como de las habilidades técnicas, pero también -y especialmente- promoviendo la suficiente capacidad para aprender a saber, aprender a aprender y aprender a trabajar en equipo (Imbernon, 2000).

\section{Pilares básicos de la enseñanza superior en España tras la implantación de la Ley Orgánica 4/2007}

\subsection{Sistema de Créditos Europeos: ECTS}

Uno de los elementos identificadores del nuevo EEES es el Sistema Europeo de Transferencia de Créditos (European Credits Transfer System, ECTS). Se entiende por "crédito" la unidad de referencia sobre la que se estructuran y organizan los currículos formativos en la mayor parte de los países y que permite medir el trabajo que debe realizar el alumnado para adquirir los conocimientos, capacidades y destrezas necesarios a fin de superar las diferentes materias de su plan de estudio.

De este modo, mientras en el modelo anterior se le atribuía al crédito una función en horas de docencia del profesor, las que variaban según fueran estas teóricas o prácticas, y no se tenía en consideración el trabajo realizado por el alumnado para la preparación de la materia fuera del horario lectivo, en tanto que los créditos ECTS agrupan el trabajo global que exige la preparación de la materia, las clases presenciales y el tiempo de estudio.

Así, el estudiante que se matricula en un curso completo tendrá que cursar 60 ECTS, correspondiéndose cada crédito con 25/30 horas, según sean estas teóricas o prácticas, que se reparten aproximadamente a lo largo de 40 semanas, dedicándole cada semana unas 40 horas. En definitiva, estamos hablando de que un alumno universitario necesita entre 1.500 y 1.800 horas de dedicación para un curso académico. 
En este esquema, si para el alumnado esta transformación supone un cambio conceptual muy significativo, para el profesorado las reformas han implicado un nuevo concepto de la enseñanza universitaria, no solo para preparar sus horas presenciales de aula, tanto teóricas como prácticas, sino también por el cambio del concepto de "tutoría" individual y grupal que este sistema trae consigo (Bozu y Canto, 2009).

\subsection{Los planes de estudio: organización y estructura}

Según el programa de Bolonia, los planes de estudio se estructuran en tres ciclos: grado, máster y doctorado, todos ellos actualmente presentes en los países firmantes de dicha declaración y similar a la ya existente en otros tantos de América Latina.

- Grado. El grado en España tiene una duración de cuatro cursos académicos para la mayoría de sus títulos, con excepción de aquellos regulados por la Comunidad Europea (como es el caso de las Ciencias de la Salud, o bien, de alguna carrera técnica) que pueden tener cinco o seis años, es decir, 260 créditos teórico-prácticos ECTS, que han de favorecer la adquisición de competencias genéricas, básicas y transversales que darán acceso al mercado laboral.

- Máster. Se busca aquí un mayor desarrollo académico, disciplinar e interdisciplinar, especialización científica de orientación hacia la investigación, o bien, de formación profesional más cualificada. Tiene una duración mínima de 60 y una máxima de 120 créditos ECTS.

- Doctorado. Tiene un papel fundamental entre el EEES y el Espacio Europeo de Investigación, ya que son los doctores quienes articulan la innovación y la investigación. Queda regulado, como se dijo anteriormente, por el RD 99/2011 como complemento del RD 1393/2007, modificado por el RD 861/2010, instrumentos que normalizan los títulos de grado y máster. El principal cambio reside, posiblemente, en que antes estos programas incluían una etapa de formación después de la licenciatura, en cambio hoy el acceso parte desde la base que el estudiante cumpla ya con unos requisitos formativos previos, aunque se prevé la posibilidad de 
que pueda realizar o no determinados complementos formativos que no requieren la estructura de ECTS. Tiene como objetivo la capacitación del alumnado y una formación avanzada en técnicas e investigación.

\section{La acreditación y la certificación}

Debido a la necesidad de aumentar la credibilidad de las instituciones universitarias a nivel europeo y nacional, después de la Declaración de Praga de 2001 se anima a las universidades e instituciones de educación superior a trabajar en buenas prácticas y difundirlas, así como a diseñar escenarios para una aceptación mutua de mecanismos de evaluación/certificación. En el 2001 en España (III Foro de Almagro) se pone de manifiesto la necesidad de establecer vínculos entre los procesos de autoevaluación y acreditación/certificación y, en consecuencia, además de las agencias de calidad ya existentes (la Catalana y la Andaluza), se crea la Agencia Gallega en 2001 y en 2002 la Agencia Nacional de Evaluación de la Calidad y Acreditación (ANECA), convertiéndose en la entidad que orientará toda la política de calidad en España (Muñoz, 2008) otorgándole, en un principio, la competencia exclusiva de acreditación de las enseñanzas oficiales que posteriormente sería asumida también por otras agencias autónomas. En la actualidad existen diez agencias que gestionan programas orientados hacia la evaluación de los solicitantes, figuras de profesor universitario, verificación de las propuestas de los planes de estudio diseñados en consonancia con el EEES, y valoración para la renovación de la acreditación inicial de los títulos, así como la obtención de sellos europeos, el seguimiento anual del título oficial para comprobar su correcta implantación y resultados, crear sistemas de evaluación del profesorado universitario, orientar a los centros universitarios en el diseño de sistemas de garantía de calidad, o bien evaluar y certificar los programas de doctorado que optan a una mención hacia la excelencia.

Hemos de decir que si bien hasta estos momentos los títulos constaban de materias, descriptores, créditos y áreas de conocimiento, esta estructura desaparece y se establece un nuevo procedimiento para la inclusión de títulos en el registro de universidades, centros y títulos en el que el plan de estudios ha de ser entendido como un contrato 
entre la universidad y la sociedad y que, por tanto, los criterios para el registro y evaluación de una titulación está en consonancia con la garantía de la calidad en el EEES.

El nuevo marco de educación superior y las directrices marcadas obligan a las universidades a tener sus propios sistemas de calidad acordes con las directrices para la elaboración de títulos universitarios recogidos en la legislación indicada anteriormente y desarrolladas en los programas de verificación y acreditación de las agencias autónomas y es, en este contexto, donde cobra importancia la evaluación de las guías docentes en tanto son las canalizadoras de su acción.

Los elementos por verificar y posteriormente acreditar de manera general son los siguientes:

1. Descripción del título.

2. Justificación.

3. Competencias (básicas, específicas, transversales).

4. Acceso y admisión de estudiantes.

5. Planificación de las enseñanzas.

6. Personal académico.

7. Recursos materiales y servicios.

8. Resultados previstos.

9. Sistema de garantía de calidad.

10. Calendario de implantación.

\section{La guía docente en el EEES}

La legislación establece los elementos que debe tener el plan de estudio, debiendo aportar información, como por ejemplo denominación de la materia, número de créditos ECTS, carácter (básico, obligatorio, optativo, trabajo de fin de grado y prácticas, entre otras), duración y ubicación temporal, competencias y su concreción en resultados de aprendizaje, actividades formativas y metodologías docentes, sistemas de evaluación acerca de los resultados de aprendizaje, contenidos y lenguas en las que se imparte. 
La Universidad de A Coruña, a tenor de las propuestas del EEES, introduce las Guías de Armonización de la Docencia Universitaria (GADU), un documento donde se visualizan los principios básicos en los que se va a fundamentar el desarrollo de cada materia y que consta de los siguientes apartados: datos identificativos, competencias de la titulación, resultados de aprendizaje, contenidos, planificación, metodología, atención personalizada, evaluación, recomendaciones y fuentes de información, todo ello en línea con los elementos nombrados anteriormente.

\subsection{Las competencias en el contexto universitario}

El diseño sobre la base de competencias es el eje en el que se desarrollan los procesos de enseñanza-aprendizaje en este nuevo marco y en el que se construyen las guías o proyectos docentes de cada una de las materias de cada título.

Son múltiples y variadas las definiciones que se dan de las competencias. Según Gimeno-Sacristán (2008), por ejemplo, estas se usan para denominar los objetivos de los programas educativos, entender y desarrollar el currículo, dirigir la enseñanza, organizar actividades de aprendizaje de los estudiantes y orientar la evaluación del alumnado. Para Carreras y Perrenoud (2008), en tanto, las competencias no solo dependen del ámbito en donde ellas se empleen, del contexto cultural o del contexto lingüístico, sino también dentro del mismo contexto pueden ser entendidas de diferentes maneras: en EE.UU. se denominan "habilidades básicas"; en el Reino Unido se les llama "habilidades centrales o troncales" o "genéricas", en el caso de Canadá. En el desarrollo del EEES ha tenido una gran incidencia el proyecto Tunning Educational Structures in Europe (2003) que combina atributos que atañen al saber, saber hacer, saber estar y saber ser, además de habilidades o destrezas.

Ahora bien, si algún sentido tiene el término de "competencia" es el lograr alumnos competentes en el mercado laboral; de hecho Sobrado (2010) las utiliza como sinónimo de nivel de capacitación, conocimientos, saberes, actitudes, capacidades y habilidades que tienen las personas y que dominan o deben dominar, o bien como señala 
Montero (2010), el resultado final ha de centrarse en la formación de alumnos competentes "capaces no solo de acumular conocimientos, sino sobre todo, de saber transmitir esos conocimientos y especialmente de aplicarlos con una finalidad laboral concreta" (p. 27).

En cuanto a la clasificación de las competencias, esta depende del enfoque o modelo del cual partamos, especialmente si nos referimos a competencias profesionales. De este modo existe una categoría referida a las competencias clave, transversales o genéricas, las cuales normalmente se definen como comunes a una rama profesional (por ejemplo, salud, ingeniería, educación) o a todas las profesiones como la base común de la profesión. Si hacemos referencia al ámbito universitario, serían aquellas comunes a la mayoría de las titulaciones. Este tipo de competencias son susceptibles de emplearse en situaciones diferentes por el alumnado o profesional respectivo, permitiendo la trasferencia de un contexto a otro (Sobrado, 2010), y suelen clasificarse en instrumentales, personales o interpersonales y sistémicas. Las primeras (instrumentales) son aquellas que miden las capacidades y la formación del titulado, por lo tanto constituyen un medio para obtener un determinado fin; las segundas (personales) medirían las habilidades de relación social y de integración en diferentes colectivos, así como la capacidad de trabajar en equipos específicos y multidisciplinares, esto es, se trata de las capacidades que permiten que las personas tengan interacción con los demás; por último, las sistémicas miden las cualidades individuales y la motivación a la hora de trabajar, ya que exigen destrezas relacionadas con la comprensión de la totalidad como un sistema.

Otra categoría es la referida a las competencias específicas que son más restrictivas en su utilización y aplicación (áreas de especialización) y constituyen la base de la profesión. Si hacemos referencia al ámbito universitario, serían aquellas propias de un ámbito o titulación, orientadas a la consecución de un perfil específico del graduado. Este tipo de competencias se divide en tres clases: académicas, disciplinares y del ámbito profesional.

En esta línea, Martínez y Echeverría (2009) nos ofrecen una clasificación en la que incluyen las competencias actitudinales: las 
académicas o relativas a conocimientos teóricos (saber); las disciplinares o el conjunto de conocimientos prácticos requeridos para cada sector profesional (hacer); las del ámbito profesional, procedimentales o instrumentales incluyen tanto las habilidades de comunicación e indagación como el know how aplicados al ejercicio de una profesión concreta (saber hacer); y por último las actitudinales (las del ser), relacionadas en cierto modo con las sistémicas y las personales.

Las competencias básicas señaladas por otros autores en particular están referidas a las capacidades intelectuales indispensables para el aprendizaje de una profesión. Este tipo de competencias se divide en tres clases: cognitivas, técnicas y metodológicas, muchas de las cuales son adquiridas en los niveles educativos previos.

\subsection{Metodología de trabajo}

Son numerosos los modelos que las universidades españolas han adoptado para desplegar estrategias que permitan el desarrollo y adquisición de las competencias sobre la base de una estructura temporal y estructural de las materias. Así se ha pasado de una distribución de materias cuatrimestrales o anuales a una estructura enteramente cuatrimestral; se organizan los cursos en dos cuatrimestres y se estructuran las materias potenciando las prácticas en grupos de estudiantes que permitan una mayor atención y seguimiento de su aprendizaje, bajo el axioma de "aprender a aprender". En la Universidad A Coruña se ha optado por impartir la materia en torno a dos tipos de agrupaciones de alumnos: unas en las que los agrupamientos son con todos los alumnos del grupo-clase y el desarrollo de las mismas permite, fundamentalmente, la exposición y explicación, por parte del profesor, de las partes que se podrían denominar genéricamente "aspectos conceptuales" de la asignatura. En las otras, los grupos de estudiantes son reducidos para poder realizar problemas, cuestiones o trabajos propuestos. Así, dentro del horario de preparación de una materia tiene un peso fundamental el trabajo autónomo del alumnado, bien sea este individual o en equipo. Al margen de estos procesos hay otras técnicas de menor índole como el aprendizaje colaborativo, el foro virtual, la presentación oral, la prueba mixta, trabajos tutelados, portafolios del alumno, debate virtual, etc. 
(Rodríguez, 2011), integrando las tecnologías para lograr una mayor comunicación e implicación de los estudiantes con el profesorado. El uso de la plataforma MOODLE ${ }^{3}$ está orientado a ese fin.

\subsection{Atención personalizada}

Uno de los elementos de calidad tiene que ver con la satisfacción de los usuarios, por lo que cualquier sistema, norma o modelo la incluye como claro exponente en su organización. En el caso de los estudiantes son dos los elementos que así la identifican: aquellos relacionados con los procesos de enseñanza-aprendizaje y, otro, no menos importante, que es la atención. Bajo este último prisma cobra especial relevancia la "tutoría", la que podemos considerar un recurso muy importante en la formación inicial y continua del alumnado, porque permite un acercamiento a un proceso educativo individualizado que facilita espacios y tiempos que no siempre se encuentran en la clase ordinaria y que son imprescindibles para mejorar la calidad de la enseñanza (De Miguel, 2006).

En este sentido las instituciones no solo deben controlar, revisar y mejorar la efectividad de los planes de acción tutorial que se implantan en los centros para ayudar al estudiante a lo largo de su carrera, sino también las tutorías individualizadas que desarrolla cada profesor respecto de su materia. No debemos olvidar dentro de esa visión de calidad total, la efectividad de los servicios de apoyo institucionales que tiene cada universidad para sus estudiantes.

\subsection{Evaluación}

La evaluación se afronta como un elemento más del proceso de enseñanza-aprendizaje del EEES, donde tanto el alumno como el docente pueden reflexionar y realizar propuestas de mejora de los procesos que van teniendo lugar en el aula, desterrando el carácter coercitivo que tradicionalmente le ha acompañado (Brown, 2003; López, 2005). Ha pasado a ser más continuada, donde intervienen

Moodle es una plataforma de aprendizaje diseñada para proporcionar a educadores, administradores y estudiantes un sistema integrado único, robusto y seguro para crear ambientes de aprendizaje personalizados. 
las metodologías programadas por el profesor en la guía, y donde el alumnado hace su propia evaluación, tanto de forma individual como grupal, de su propia implicación en el aula, de los trabajos realizados, pero también del proceso educativo en su globalidad.

La evaluación cobra un interés en sí misma como indicador de la evolución curricular de los alumnos, y por su poder como indicador institucional, pues permite valorar la efectividad de las enseñanzas en general, y del apoyo que se le ofrece al estudiante.

\subsection{Fuentes documentales}

La idea fundamental es que el alumnado vaya elaborando su propia base de fuentes bibliográficas. Para ello se le proporciona abundante información y fuentes documentales, lecturas obligatorias y voluntarias. Por otra parte, es el propio estudiante quien tiene que hacer investigaciones acerca de aspectos concretos de la temática, ya sea por sugerencias del profesorado, o bien, por propia iniciativa, para el enriquecimiento y adecuación a sus intereses.

\section{Conclusión}

En un intento de sintetizar los elementos que singularizan las reformas que afectan a la universidad en la creación del EEES podemos concluir diciendo que el proceso de Bolonia nos conduce a una mayor compatibilidad y comparabilidad de los sistemas de educación superior, facilita la movilidad de los estudiantes y la adopción de los criterios y directrices para la garantía de calidad en el EEES. También se ha creado un registro europeo de agencias de calidad y se ha establecido el Marco Europeo de Calificaciones que permite su descripción en términos de volumen de trabajo, nivel, resultados de aprendizaje, competencias y perfil (Gallardo y Reyes, 2010; Rodríguez, 2011). Además, promueve el suplemento para el diploma y para el Sistema Europeo de Transferencia y Acumulación de Créditos (ECTS) en los que el alumnado, a priori, es el centro de referencia de todo el proceso de enseñanza-aprendizaje, así como nuevas metodologías docentes y de evaluación, el uso de las TIC, potenciación del trabajo autónomo y en equipo, y una nueva concepción del 
proceso de enseñanza-aprendizaje universitario, que van unidos a la reestructuración de los estudios universitarios (enseñanzas de grado, máster y doctorado) y de los planes de estudios (materias de formación básica, obligatorias, optativas, prácticas externas y trabajo de fin de grado), además de cambios en las formas de organización de los centros universitarios. En este sentido, la guía docente ayuda al alumnado y al propio profesor, porque permite tener una propuesta clara del diseño y desarrollo de la materia. De ahí la necesidad de socializar y legitimar ante los profesores las demandas estudiantiles respecto de la docencia, pues es probable que este desconocimiento explique muchos desencuentros entre ambos estamentos (Asún, Zúñiga y Ayala, 2013).

Fundamentalmente, el EEES desde su estructura formativa y educadora debería ser capaz de crear profesionales competentes, cultos, responsables, reflexivos, críticos y con capacidad de adaptar y conectar de una forma más clara el periodo académico con las necesidades futuras en el mercado laboral.

Como resumen de lo expuesto diremos que las dificultades e inconvenientes que presenta este sistema habrán merecido la pena si redundan en un mejor aprendizaje para los alumnos.

\section{Referencias}

Agencia Nacional de Evaluación de la Calidad y Acreditación (ANECA) (2004). La adecuación de las titulaciones de maestro al Espacio Europeo de Educación Superior. Recuperado el día 25 de marzo de 2014 desde http://www. aneca.es/var/media/150424/ibroblanco_jun05_documentacion.pdf.

Asún, R., Zúñiga, C. y Ayala, M.C. (2013). La formación por competencias y los estudiantes: confluencias y divergencias en la construcción del docente ideal. Revista Calidad en la Educación (38), 277-304. Recuperado el día 27 de marzo de 2014, de http://www.scielo.cl/scielo. php?script=sci_arttext\&pid=S0718-45652013000100008\&lng=es\&t lng=es. 10.4067/S0718-45652013000100008.

Bozu, Z. y Canto, P.J. (2009). El profesorado universitario en la sociedad del conocimiento: competencias profesionales docentes. Revista de Formación e Innovación Educativa Universitaria, 2(2), 87-97. 
Brown, S. (2003). Estrategias institucionales en evaluación. En Browny, S. y Glasner, A. (Eds.), traducción de Miguel Callizo, Evaluar en la universidad. Problemas y nuevos enfoques (pp. 23-34). Madrid: Narcea Ediciones.

Carreras, J. y Perrenoud, P. (2008). El debate sobre las competencias en la enseñanza universitaria. Barcelona: ICE y Ediciones Octaedro.

Declaración de Bolonia (1999). El Espacio Europeo de Educación Superior. Declaración conjunta de los Ministros Europeos de Educación. Bolonia, 19 de junio de 1999.

Declaración de la Sorbona (1998). Declaración conjunta para la armonización del diseño del Sistema de Educación Superior Europeo. París, 25 de mayo de 1998.

De Miguel, M. (2006). Modalidades de enseñanza centradas en el desarrollo de competencias: orientaciones para promover el cambio metodológico en el Espacio Europeo de Educación Superior (EEES). Oviedo: Universidad de Oviedo.

Delgado, A.M., Borge, R., García, J., Oliver, R. y Salomón, L. (2005). Competencias y diseño de la evaluación continua y final en el Espacio Europeo de Educación Superior. Madrid: Ministerio de Educación y Ciencia.

Fernández-Salinero, C. (2006). Las competencias en el marco de la convergencia europea: un nuevo concepto para el diseño de programas educativos. Encounters on Education, 7, 131-153.

Gallardo, G. y Reyes, P. (2010). Relación profesor-alumno: arista fundamental para el aprendizaje. Revista Calidad de la Educación (32), 78-108.

Gimeno-Sacristán, J. (2008). Diez tesis sobre la aparente utilidad de las competencias en educación. En Gimeno, J. (Comp.), Educar por competencias, ¿qué hay de nuevo? (pp. 15-58). Madrid: Morata.

González, C. (2010). El aprendizaje y el conocimiento académico sobre la enseñanza como claves para mejorar la docencia universitaria. Revista Calidad en la Educación (33), 123-146.

Imbernon, F. (2000). Un nuevo profesorado para una nueva universidad: ¿conciencia o presión? Revista Interuniversitaria de Formación del Profesorado (38), 37-46.

Knight, P. (2005). El profesorado de educación superior: formación para la excelencia. Madrid: Narcea Ediciones.

Ley Orgánica 4/2007, de 12 de abril, por la que se modifica la Ley Orgánica 6/2001, de 21 de diciembre, de universidades. «BOE» núm. 89, de 13 de abril de 2007, pp. 16.241 a 16.260. 
334 EL PROYECTO DOCENTE EN LA UNIVERSIDAD ESPAÑOLA SEGÚN EL ESPACIO

EUROPEO DE EDUCACIÓN SUPERIOR - J. Muñoz- Cantero y M. Mato- Vásquez

Levy-Leboyer, C. (2000). Gestión de competencias. Barcelona: Ediciones Gestión.

López, F. (2005). Metodología participativa en la enseñanza universitaria. Madrid: Narcea Ediciones.

Malheiro, X.M. (2011). Proxecto docente. A Coruña: UDC.

Martínez, P. y Echeverría, B. (2009). Formación basada en competencias. Revista de Investigación Educativa, 27, 125-147.

Montero, M. (2010). El proceso de Bolonia y las nuevas competencias. Tejuelo, 9, 19-37.

Muñoz, J. M. (2008). Garantía de calidad en el Espacio Europeo de Educación Superior. En Exxeberría Sagastume, F. et al. (Eds.). Convivencia, equidad, calidad (pp. 45-70). Domostia: Eirem.

Muñoz, J. M., Rebollo, N. y Espiñeira, E. (2013). Desarrollo, dominio y relevancia de competencias en el grado de educación social. Revista Galego Portuguesa de Psicoloxía e Educación (21), 227-247.

Rodríguez, M. (2011). Metodologías docentes en el EEES: de la clase magistral al portafolio. Tendencias pedagógicas (17), 83-102.

Sobrado, L. (2010). Competencias (profesionales, académicas). En Caride Gómez, J. A. y Trillo Alonso, F. (Eds.). Dicccionario Galego de Psicopedagoxía. Santiago de Compostela: Galaxia.

Triadó, X., Aparicio, P. y Elasri, A. (2013). La evaluación de competencias en la educación superior: el caso de un máster universitario. REIRE, Revista d'Innovació i Recerca en Educació, 6(1), 34-52. Recuperado el día 27 de marzo del 2014, de: http://www.ub.edu/ice/reire.htm

Tunning Educational Structures in Europe (2003). Informe final. Bilbao (España): Universidad de Deusto.

Villa, A. y Villa, O. (2007). El aprendizaje basado en competencias y el desarrollo de la dimensión social en las universidades. Educar, 40, $15-48$.

Recibido: 08/05/2014

Aceptado: 20/05/2014 